\title{
Editorial: Multiscale Lattices and Composite Materials: Optimal Design, Modeling and Characterization
}

\author{
Fernando Fraternali ${ }^{1 *}$, Chiara Daraio ${ }^{2}$ and Julian Rimoli ${ }^{3}$ \\ ${ }^{1}$ Department of Civil Engineering, University of Salerno, Fisciano, Italy, ${ }^{2}$ Division of Engineering and Applied Science, \\ California Institute of Technology, Pasadena, CA, United States, ${ }^{3}$ School of Aerospace Engineering, Georgia Tech, Atalnta, \\ GA, United States
}

Keywords: lattice materials, mechanical metamaterials, innovative composites, multiscale mechanics, structural health monitoring, hyperspectral imaging

\section{Editorial on the Research Topic}

\section{Multiscale Lattices and Composite Materials: Optimal Design, Modeling and Characterization}

\section{OPEN ACCESS}

Edited by:

Nicola Maria Pugno, University of Trento, Italy

Reviewed by:

Marco Miniaci,

Swiss Federal Laboratories for Materials Science and

Technology, Switzerland

*Correspondence:

Fernando Fraternal

f.fraternali@unisa.it

Specialty section:

This article was submitted to

Mechanics of Materials,

a section of the journal

Frontiers in Materials

Received: 20 May 2019 Accepted: 05 August 2019

Published: 20 August 2019

Citation:

Fraternali F, Daraio C and Rimoli J (2019) Editorial: Multiscale Lattices and Composite Materials: Optimal

Design, Modeling and

Characterization. Front. Mater. 6:199.

doi: 10.3389/fmats.2019.00199
The Research Topic "Multiscale lattices and composite materials:" (MLCM) is focused on the optimal design, modeling, and characterization of novel lattices, composite materials, and structures at different scales, through the control of the internal architecture of the system.

A fundamental goal of this article collection is the study of mechanical metamaterials that are able to form next-generation-generation cellular solids; lattice materials, multiscale composites; and structural-scale systems. The collection took inspiration from the peculiar behaviors exhibited by structured materials at multiple scales (Bosia et al., 2018). The latter include, for example, high stiffness, strength, and toughness at extremely low densities (Meza et al., 2014), phononic bandgaps (Lu et al., 2009), sound control ability (Cummer et al., 2016); negative effective mass density (Liu et al., 2000); localized confined waves (Theocharis et al., 2013), to name but a few examples. The research reported devoted special attention to the creation of complex mechanical systems with properties derived mainly from their geometric design rather than their chemical composition (Cummer et al., 2016; Bertoldi et al., 2017). Also investigated was the use of multiscale lattices to optimally design reinforcing elements for novel composite materials (Fleck et al., 2010; Li et al., 2014). The chosen modeling and experimental approaches were able to predict and characterize the intrinsically complex mechanical behavior of the analyzed systems through multiscale techniques.

The papers forming the MLCM collection can be grouped into two basic categories. The first of these is centered around the design, modeling, and characterization of lattice structures at different scales, through the maximization of the frequency bandgap width at suitable center frequencies (Arretche and Matlack; Bacigalupo et al.); the optimal design and mechanical modeling of tensegrity metamaterials (De Tommasi et al.), superstable pre-stressed networks (Kelly et al.), graphene sheets (Genoese et al.); dome-shaped auxetic metamaterials (Easey et al.); and solar façades that employ dynamic sunscreens with tensegrity architecture (Babilio et al.). This first group of papers also includes contributions dealing with the development of non-destructive testing and structural health monitoring techniques that make use of guided elastic waves (Miniaci et al.), as well as the experimental characterization of the microstructure of the Nephila dragline silk (Stehling et al.). 
A second category focuses on the modeling and characterization of novel composite materials, with emphasis on the mechanical properties, for example, of bamboo fiber-reinforced composites (Javadian et al.); the effects of defects, porosity, and damage on the mechanical properties of metallic materials to be employed in additive manufacturing processes (Goodall et al.); the macroscopic response of micropolar continua with anisotropic microstructure (Fantuzzi et al.); the addition of lattice-shaped inclusions to metaconcretes (Briccola et al.); and the propagation of pressure waves in three-dimensional arrangements of coated spheres (Dupont et al.).

\section{REFERENCES}

Bertoldi, K., Vitelli, V., Christensen, J., and Van Hecke, M. (2017). Flexible mechanical metamaterials. Nat. Rev. Mater. 2:17066. doi: 10.1038 /natrevmats.2017.66

Bosia, F., Krushynska, A. O., Miniaci, M., Morvan, B., and Pugno, N. M. (2018). Editorial: advances in mechanical metamaterials. Front. Mater. 5:56. doi: 10.3389/fmats.2018.00056

Cummer, S. A., Christensen, J., and Alu, A. (2016). Controlling sound with acoustic metamaterials. Nat. Rev. Mater. 1:16001. doi: 10.1038/natrevmat s.2016.1

Fleck, N. A., Deshpande, V. S., and Ashby, M. F. (2010). Micro-architectured materials: past, present and future. Proc. R. Soc. A 466, 2495-2516. doi: 10.1098/rspa.2010.0215

Li, J., Wu, Z., Huang, C., and Li, L. (2014). Multiscale carbon nanotube-woven glass fiber reinforced cyanate ester/epoxy composites for enhanced mechanical and thermal properties. Compos. Sci. Technol. 104, 81-88. doi: 10.1016/j.compscitech.201 4.09.007

Liu, Z., Zhang, X., Mao, Y., Zhu, Y. Y., Yang, Z., Chan, C. T., et al. (2000). Locally resonant sonic materials. Science 289, 1734-1736. doi: 10.1016/S0921-4526(03)00487-3
Our hope is that the research presented in this collection will stimulate new and exciting research in the fields of mechanical metamaterials and multiscale composite materials and structures, through an integrated approach that includes the design and the mechanical modeling of real-scale, or reducedscale prototypes; the optimal control of suitable design variables; and the experimental validation of the theoretical predictions.

\section{AUTHOR CONTRIBUTIONS}

All authors listed have made a substantial, direct and intellectual contribution to the work, and approved it for publication.

Lu, M. H., Feng, L., and Chen, Y. F. (2009). Phononic crystals and acoustic metamaterials. Mater. Today 12, 34-42. doi: 10.1016/S1369-7021(09)70315-3

Meza, L. R., Das, S., and Greer, J., R. (2014). Strong, lightweight, and recoverable three-dimensional ceramic nanolattices. Science 345, 1322-1326. doi: $10.1126 /$ science. 1255908

Theocharis, G., Boechler, N., and Daraio, C. (2013). "Nonlinear phononic structures and metamaterials," in Acoustic Matematerials and Phononic Crystals, ed P. A. Deymier (Berlin; Heidelberg: Springer-Verlag), 173.

Conflict of Interest Statement: The authors declare that the research was conducted in the absence of any commercial or financial relationships that could be construed as a potential conflict of interest.

The handling editor declared a past collaboration with the authors FF, CD.

Copyright (c) 2019 Fraternali, Daraio and Rimoli. This is an open-access article distributed under the terms of the Creative Commons Attribution License (CC BY). The use, distribution or reproduction in other forums is permitted, provided the original author(s) and the copyright owner(s) are credited and that the original publication in this journal is cited, in accordance with accepted academic practice. No use, distribution or reproduction is permitted which does not comply with these terms. 